\title{
A case of hepatocellular carcinoma with skin injury of the upper abdominal wall after transcatheter arterial chemoembolization: a case report

\author{
Hiromitsu Kanzaki*, Kazuhiro Nouso, Koji Miyahara, Naoko Kajikawa, \\ Sayo Kobayashi, Ichiro Sakakihara, Shota Iwadow, Shuji Uematsu, \\ Ryoichi Okamoto, Kunihiro Shiraga, Motowo Mizuno and Yasuyuki Araki
}

Address: Department of Internal Medicine, Hiroshima City Hospital, 7-33, Moto-machi, Naka-ku, Hiroshima-city, Hiroshima, 730-8518, Japan

Email: HK* - kanzaki@qc4.so-net.ne.jp; KN - nouso@cc.okayama-u.ac.jp; KM - m.koji.sup@ezweb.ne.jp; NK - naoko.quazi@docomo.ne.jp; SK - sayo44@nifty.com; IS - i.sakaki@nifty.com; SI - gmd15041@cc.okayama-u.ac.jp; SU - cellist@mvd.biglobe.ne.jp;

RO - okamoto@ms8.megaegg.ne.jp; KS - whitemarch@h4.dion.ne.jp; MM - mmizuno@md.okayama-u.ac.jp; YA - araki@cc22.ne.jp

* Corresponding author

Received: 12 April 2009 Accepted: 3 August 2009 Published: I September 2009

Cases Journal 2009, 2:7197 doi: 10.4076/1757-1626-2-7/97

This article is available from: http://casesjournal.com/casesjournal/article/view/7/97

(c) 2009 Kanzaki et al.; licensee Cases Network Ltd.

This is an Open Access article distributed under the terms of the Creative Commons Attribution License (http://creativecommons.org/licenses/by/3.0), which permits unrestricted use, distribution, and reproduction in any medium, provided the original work is properly cited.

\begin{abstract}
Introduction: Transcatheter arterial chemoembolization has been widely used to treat advanced hepatocellular carcinoma that cannot be treated by local ablation therapies or surgical resection. The effectiveness of transcatheter arterial chemoembolization in prolonging survival has been well established, and approximately one third of newly discovered hepatocellular carcinoma patients were repeatedly treated by transcatheter arterial chemoembolization in Japan. Various kinds of complications have been reported, and many of which are general complications such as hepatic coma, jaundice, fever-up, ascites, and bile duct injury.

The hepatic falciform artery is found frequently during postmortem anatomic dissection and the incidence of hepatic falciform artery is reported to be over $60 \%$. Hepatic falciform artery is known to be the responsible artery for supraumbilical skin rash development after arterial chemo infusion therapy; however, skin complications after transcatheter arterial chemoembolization are rare.

Case presentation: A 70-year-old female with chronic hepatitis $C$ infection was diagnosed as having hepatocellular carcinoma ( $\$ 4,20 \mathrm{~mm}$ in diameter). Transcatheter arterial chemoembolization was performed via the left hepatic artery, which was a feeding artery of the hepatocellular carcinoma. Two days after that, supraumbilical skin rash with local tenderness and redness appeared. Retrospective analysis revealed that occlusion of the hepatic falciform artery branching from the left hepatic artery with micromaterials caused the skin lesion.

Conclusion: We should keep in mind that anticancer drugs or embolic materials can flow into the HFA and may cause abdominal wall injury after transcatheter arterial chemoembolization.
\end{abstract}




\section{Case presentation}

A 70-year-old Japanese female (Asian) had suffered from chronic hepatitis C since 1992. In March 2006, hepatocellular carcinoma (HCC) (20 mm in diameter) was found in liver segment 4 , and she was admitted to our hospital. She had a history of tuberculosis of the lymph nodes in 1992.

Laboratory findings on admission were as follows: white blood cell count $1.77 \times 1000 / \mathrm{mm}^{3}$, red blood cell count $339 \times 104 / \mathrm{mm}^{3}$, hemoglobin $11.3 \mathrm{~g} / \mathrm{dL}$, hematocrit $31.9 \%$, platelet count $3.1 \times 10000 / \mathrm{mm}^{3}$, total protein $7.3 \mathrm{~g} / \mathrm{dL}$, albumin $3.5 \mathrm{~g} / \mathrm{dL}$, total bilirubin $1.7 \mathrm{mg} / \mathrm{dL}$, direct bilirubin $0.7 \mathrm{mg} / \mathrm{dL}$, aspartate aminotransferase $68 \mathrm{IU} / \mathrm{L}$, alanine aminotransferase $35 \mathrm{IU} / \mathrm{L}$, alkaline phosphatase $402 \mathrm{IU} / \mathrm{L}, \gamma$-glutamyltranspeptidase $13 \mathrm{IU} / \mathrm{L}$, cholinesterase $108 \mathrm{IU} / \mathrm{L}$, total cholesterol $137 \mathrm{mg} / \mathrm{dL}$, blood urea nitrogen $16 \mathrm{mg} / \mathrm{dL}$, Creatinine $0.7 \mathrm{mg} / \mathrm{dL}$, Na $142.5 \mathrm{mEq} / \mathrm{L}$, K $3.0 \mathrm{mEq} / \mathrm{L}$, Cl $110.2 \mathrm{mEq} / \mathrm{L}$, Ca $8.8 \mathrm{mg} / \mathrm{dL}$, prothrombin time 63.8\%, ICG-R 33.8\%, ICG-K 0.072, des-gamma-carboxy prothrombin $325 \mathrm{mAU} / \mathrm{mL}$, and alpha-fetoprotein $6.8 \mathrm{ng} / \mathrm{mL}$. The patient was positive for HCV antibody but negative for hepatitis B virus surface antigen.

Enhancement in the early phase and perfusion defect in the delayed phase were observed in segment $4(20 \mathrm{~mm}$ in diameter) of the liver by computed tomography, and collateral veins were also noted (Figure 1). The tumor findings by abdominal ultrasonography were typical of HCC, which was a hyperechoic space occupied by a lesion with hallow. Hepatic arteriography showed tumor staining in the delayed phase, and the feeder of the lesion was A4 (artery 4). Lipiodol (2 ml) mixed with epirubicin (12 mg) followed by gelatin sponge was injected via the left hepatic artery, and A4 was completely occluded.

Soon after Transcatheter arterial chemoembolization (TACE), she complained of epigastric pain, and a skin

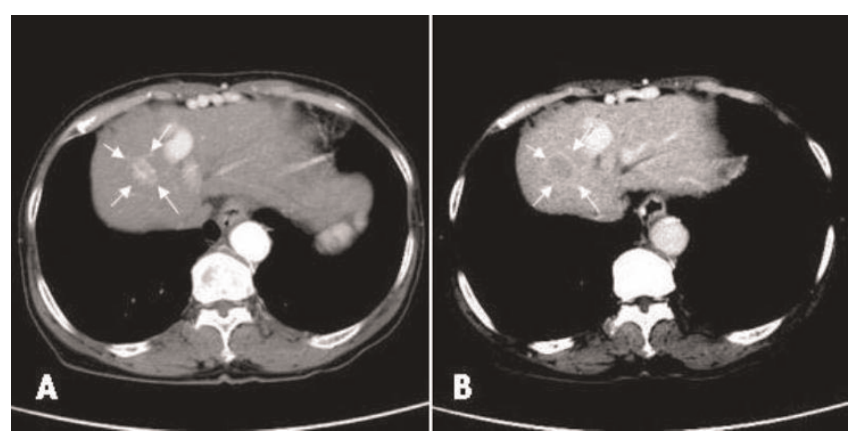

Figure I. Axial computed tomography image with contrast enhancement before angiography. Classical HCC $(20 \mathrm{~mm})$ was observed in segment 4 (arrows). Collateral veins were seen along the abdominal wall (arrow heads). A; Early phase. B; Delayed phase.

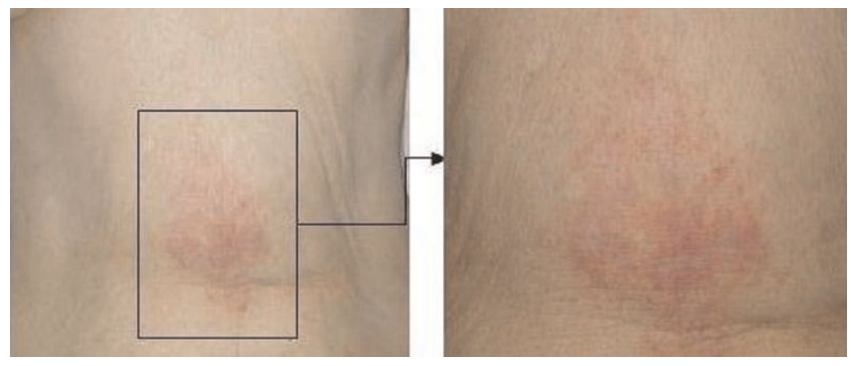

Figure 2. Skin lesion at 3 days after TACE. The patient complained of epigastric pain soon after TACE, and the pain persisted for 5 days. Skin redness appeared on the upper abdominal wall on day 2 .

rash appeared on the upper abdominal wall on day 2 (Figure 2). Angiography was retrospectively re-evaluated. The HFA branching from the left hepatic artery was faintly observed in the early arterial phase, and the flow persisted until the delayed phase (Figure 3). After TACE, hepatic falciform artery (HFA) had completely disappeared. The area of the skin lesion was consistent with the perfusion area of the HFA, so that inflow of the anticancer drug to the HFA and its subsequent occlusion were presumed to be the cause of the skin rash.

We injected steroid in the site on day 3 and 7 , and prescribed tranilast (anti-allergic agent). At 5 days after TACE, the skin rash and pain resolved, and purple spots and hard subcutaneous nodules emerged on day 7 (Figure 4). Ten months later, the skin rash had completely healed.

\section{Discussion}

The HFA arises as a small terminal branch of the left or middle hepatic artery, runs through the hepatic falciform ligament, distributes itself around the umbilicus, and communicates with branches of the superior and inferior epigastric arteries [7-13]. Michels et al. reported that the HFA is found frequently during postmortem anatomic

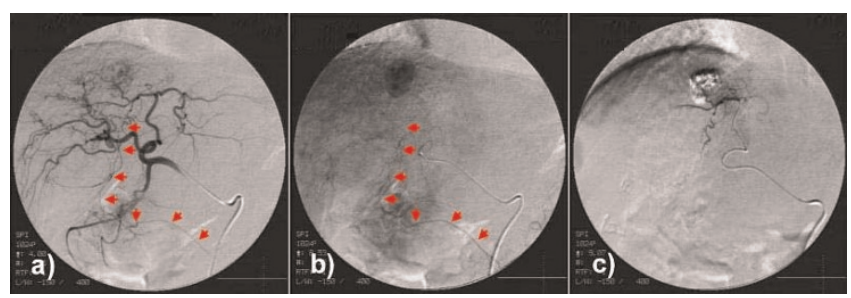

Figure 3. (A) Arterial phase of the left hepatic arteriogram showing a dilated falciform artery (arrows) arising from the left hepatic artery. (B) In the delayed phase, the flow persisted, and HCC staining was clearly detected in segment 4. (C) The HFA shadow completely disappeared after TACE. 


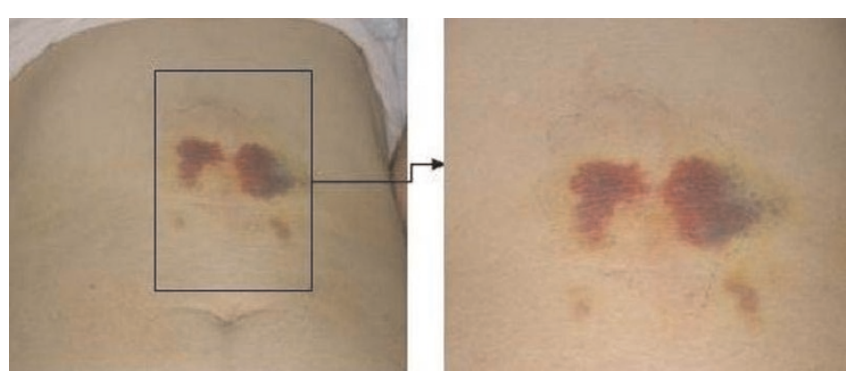

Figure 4. After the skin rash and pain were resolved, purple spot and hard subcutaneous nodule was emerged.

dissections, and that the incidence of HFA was 39 of 58 patients $(67.2 \%)$ [13]. There are some reports on skin complications in angiography caused by the inflow of anti-cancer drugs or occlusion materials to HFA [7-12]. The complication is rare and does not necessarily occur in all cases with HFA. However, once skin injury has occurred, it is sometimes severe enough to require debridement. Therefore, we should always pay attention to the artery during transcatheter arterial treatments.

There are two major factors concerning skin injury due to transcatheter therapies. One is the size of the occlusion material: small particles like Lipiodol sometimes embolize the perfusion area completely [11-12]. The other is the duration of chemotherapeutic agents: continuous arterial injection of anti-cancer drugs is known to induce skin injury [10]. We used Lipiodol mixed with epirubicin, and the dose was the same as we routinely use, so that the reason for the skin lesion seems different from the two reported factors. It is known that portal hypertension and a decrease in portal vein flow cause a compensatory increase of hepatic arterial flow and the development of portal collateral vessels [7]. Our case showed severe collateral vein accompanied by portal hypertension. Therefore, it is possible that HFA (collateral vein) flow increased, and the volume of chemical micromaterials flowing into the HFA was enhanced, resulting in skin injury.

While high incidence of HFA (67.2\%) was reported on postmortem anatomic dissections, the reported incidences of HFA on angiography were low (ranging from 2\% to $24.5 \%$ ) [7-9]. One of the reasons for these discrepancies seems to be the velocity of arterial flow. The flow was often too slow to be regularly detected during the early arterial phase on angiography [9]. The similarity of the HFA route with those of other arteries might be another reason. The HFA runs close to the hepatic artery, cystic artery, and gastroduodenal artery, so that it is easy to misinterpret the HFA.

There are controversies with regard to the relationship between the HFA and the hepatic disease state. Kim et al. reported that patients with an HFA had significantly higher serum bilirubin levels, lower albumin levels, and longer prothrombin times than patients without one. Chung et al. reported that portal vein collateral vessels were more frequently observed in patients with HFA presence than absence [6]. Conversely, Gibo et al. reported no significant difference in HFA incidence between a chronic liver disease group and normal liver group [8]. Although the mechanism of the skin injury in our case can be explained by Chung's report, further study is needed to reach a conclusion regarding these discrepancies.

For the prevention of skin rash, it is reasonable to perform TACE by placing the tip of the microcatheter beyond the origin of the HFA, or embolization of the HFA with microcoils or gelatin sponge prior to TACE. Because the HFA often communicates with branches of inferior and superior epigastric arteries, the skin lesion does not occur when embolizing the HFA with micro coils or simple gelatin sponge particles [8-12]. Hence, it is reasonable to consider embolizing the HFA with micro coils to prevent such a complication, especially in cases with severe portal hypertension or undergoing long-term chemotherapy with a reserved microcatheter, in which the drug volume needs to be increased.

In conclusion, we should keep in mind that anticancer drugs or embolic materials can flow into the HFA and may cause abdominal wall injury after TACE.

\section{Abbreviations}

HCC, hepatocellular carcinoma; HFA, hepatic falciform artery; TACE, transcatheter arterial chemoembolization.

\section{Consent}

Written informed consent was obtained from the patient for publication of this case report and accompanying images. A copy of the written consent is available for review by the journal's Editor-in-Chief.

\section{Competing interests}

The authors declare that they have no competing interests.

\section{Authors' contributions}

The main author is $\mathrm{HK}$. $\mathrm{KN}$ support to write this manuscript. KM, NK, SK, IS, SI, SU, RO, KS, MM, YA belongs to liver section and treat the patient together, corrected patient's data and gave a critical suggestion for making this manuscript. All authors read and approved the final manuscript.

\section{References}

I. Josep M Llovet: Updated treatment approach to hepatocellular carcinoma. J Gastroenterol 2005, 40:225-235.

2. Ikai I, Arii S, Ichida T, Okita K, Omata M, Kojiro M, Takayasu K, Nakanuma Y, Makuuchi M, Matsuyama Y, Yamaoka Y: Report of the 
16th follow-up survey of primary liver cancer. Hepatol Res 2005, 32:163-172

3. Kiely JM, Rilling WS, Touzios JG, Hieb RA, Franco J, Saeian K, Quebbeman EJ, Pitt HA: Chemoembolization in patients at high risk: results and complications. J Vasc Interv Radiol 2006, 17:47-53.

4. Kim HK, Chung YH, Song BC, Yang SH, Yoon HK, Yu E, Sung KB, Lee YS, Lee SG, Suh DJ: Ischemic bile duct injury as a serious complication after transarterial chemoembolization in patients with hepatocellular carcinoma. I Clin Gastroenterol 200I, 32:423-427.

5. Matsumoto K, Nojiri J, Takase Y, Egashira Y, Azama S, Kato A, Kitahara K, Miyazaki K, Kudo Sho: Cerebral lipiodol embolism: A complication of transcatheter arterial chemoembolization for hepatocellular carcinoma. Cardiovasc Intervent Radiol 2007, 30:5|2-5|4.

6. Chung JW, Park JH, Han JK, Choi BI, Han MC, Lee HS, Kim CY: Hepatic tumors: Predisposing factors for complications of transcatheter oily chemoembolization. Radiology 1996, 198: 33-40.

7. Kim DE, Yoon HK, Ko GY, Kwon JS, Song HY, Sung KB: Hetpatic Falciform Artery is prophylactic embolization needed before short-term hepatic arterial chemoinfusion? Am J Roentgenol 1999, 172:1597-1599.

8. Baba $\mathrm{Y}$, Miyazono N, Ueno K, Kanetsuki I, Nishi H, Inoue H, Nakajo M: Hepatic falciform artery. Angiographic finding in 25 patients. Acta Radiologica 2000, 41:329-333.

9. Gibo M, Hasuo K, Inoue A, Miura N, Murata S: Hepatic falciform artery: angiographic observations and significance. Abdom Imaging 2001, 26:515-519.

10. Williams DM, Cho KJ, Ensminger WD, Ziessman HA, Gyves JW: Hepatic Falciform Artery: Anatomy, Angiographic Appearance, and Clinical Significance. Radiology 1985, 156:339-340.

II. Lin CC, Wu DK, Shih PM, Liu GC, Chuang WL: Supraumbilical skin rash and fat necrosis after transcatheter arterial chemoembolization: a case report. Kaohsiung J Med Sci 2004, 20:36-40.

12. Umemura T, Yamamura N, Nagata A, Shibata A, Yamashita K, Ohata T, Yamada T, Katsuyama T, Kiyosawa K: Case report: Steatonecrosis in the upper abdomen following transcatheter arterial embolization for hepatocellular carcinoma. J gastroenterol Hepatol 1998, 13:47|-474.

13. Michels NA: Collateral arterial pathways to the liver after ligation of the hepatic artery and removal of the celiac axis. In Blood supply and anatomy of the upper abdominal organs. Edited by Michels NA. Philadelphia: JB Lippincott; 1955:317-318.

\section{Do you have a case to share?}

Submit your case report today

- Rapid peer review

- Fast publication

- PubMed indexing

- Inclusion in Cases Database

\section{Any patient, any case, can teach us something}

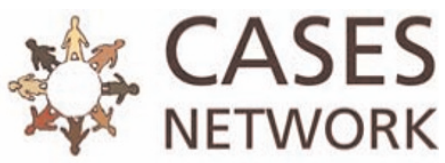

www.casesnetwork.com 